\title{
Safeguarding Stem Cell-Based Regenerative Therapy against Iatrogenic Cancerogenesis: Transgenic Expression of DNASE1, DNASE1L3, DNASE2, DFFB Controlled By POLA1 Promoter in Proliferating and Directed Differentiation Resisting Human Autologous Pluripotent Induced Stem Cells Leads to their Death
}

\author{
Marek Malecki ${ }^{1,2 *}$, Christine LaVanne ${ }^{2}$, Dominique Alhambra ${ }^{2}$, Chaitanya Dodivenaka ${ }^{3}$ Sarah Nagel $^{3}$ and Raf Malecki ${ }^{1,4}$ \\ ${ }^{1}$ Phoenix Biomolecular Engineering Foundation, San Francisco, CA 94105, USA \\ 2University of Wisconsin, Madison, WI 53706, USA \\ ${ }^{3}$ South Dakota State University, Brookings, SD 57007, USA \\ ${ }^{4}$ San Francisco State University, San Francisco, CA 94123, USA
}

\begin{abstract}
Introduction

The worst possible complication of using stem cells for regenerative therapy is iatrogenic cancerogenesis. The ultimate goal of our work is to develop a self-triggering feedback mechanism aimed at causing death of all stem cells, which resist directed differentiation, keep proliferating, and can grow into tumors.

Specific aim

The specific aim was threefold: (1) to genetically engineer the DNA constructs for the human, recombinant DNASE1, DNASE1L3, DNASE2, DFFB controlled by POLA promoter; (2) to bioengineer anti-SSEA-4 antibody guided vectors delivering transgenes to human undifferentiated and proliferating pluripotent stem cells; (3) to cause death of proliferating and directed differentiation resisting stem cells by transgenic expression of the human recombinant DNases (hrDNases).

\section{Methods}

The DNA constructs for the human, recombinant DNASE1, DNASE1L3, DNASE2, DFFB controlled by POLA promoter were genetically engineered. The vectors targeting specifically SSEA-4 expressing stem cells were bioengineered. The healthy volunteers' bone marrow mononuclear cells (BMMCs) were induced into human, autologous, pluripotent stem cells with non-integrating plasmids. Directed differentiation of the induced stem cells into endothelial cells was accomplished with EGF and BMP. The anti-SSEA 4 antibodies' guided DNA vectors delivered the transgenes for the human recombinant DNases' into proliferating stem cells.

\section{Results}

Differentiation of the pluripotent induced stem cells into the endothelial cells was verified by highlighting formation of tight and adherens junctions through transgenic expression of recombinant fluorescent fusion proteins: VE cadherin, claudin, zona occludens 1 , and catenin. Proliferation of the stem cells was determined through highlighting transgenic expression of recombinant fluorescent proteins controlled by POLA promoter, while also reporting expression of the transgenes for the hrDNases. Expression of the transgenes for the DNases resulted in complete collapse of the chromatin architecture and degradation of the proliferating cells' genomic DNA. The proliferating stem cells, but not the differentiating ones, were effectively induced to die.

Conclusion

Herein, we describe attaining the proof-of-concept for the strategy, whereby transgenic expression of the genetically engineered human recombinant DNases in proliferating and directed differentiation resisting stem cells leads to their death. This novel strategy reduces the risk of iatrogenic cancerogenesis in stem cell therapy.
\end{abstract}

Keywords: Regenerative medicine; Stem cell therapy; Neoplasm; Stem cell induced cancerogenesis; Pluripotent stem cell; DNase; DNA polymerase; Stage specific embryonal antigen

\section{Introduction}

Human pluripotent stem cells are the foundations for regenerating organs injured by disease, reconstructing tissues damaged by iatrogenic effects of therapies, rejuvenating systems affected by aging, and correcting congenital disorders caused by genetic mutations [1-4]. However, using these cells carries a risk of cancerous transformation of the therapeutically-introduced human pluripotent stem cells [5-12].

Various strategies were recently developed to reduce the risk of iatrogenic cancerogenesis. Genetically engineered and synthetic
*Corresponding author: Marek Malecki MD PhD, Phoenix Biomolecular Engineering Foundation, San Francisco, CA, 94105-191111, USA, Tel: 4157134370; Fax: 4157134371; Skype: mm-pbmef; E-mail: mm@pbmef.org

Received May 03, 2013; Accepted July 14, 2013; Published July 22, 2013

Citation: Malecki M, Vanne CL, Alhambra D, Dodivenaka C, Nagel S, et al. (2013) Safeguarding Stem Cell-Based Regenerative Therapy against latrogenic Cancerogenesis: Transgenic Expression of DNASE1, DNASE1L3, DNASE2, DFFB Controlled By POLA1 Promoter in Proliferating and Directed Differentiation Resisting Human Autologous Pluripotent Induced Stem Cells Leads to their Death. J Stem Cell Res Ther S9: 005e. doi:10.4172/2157-7633.S9-005 [PubMed]

Copyright: ( 2013 Malecki M, et al. This is an open-access article distributed under the terms of the Creative Commons Attribution License, which permits unrestricted use, distribution, and reproduction in any medium, provided the original author and source are credited. 
Citation: Malecki M, Vanne CL, Alhambra D, Dodivenaka C, Nagel S, et al. (2013) Safeguarding Stem Cell-Based Regenerative Therapy against latrogenic Cancerogenesis: Transgenic Expression of DNASE1, DNASE1L3, DNASE2, DFFB Controlled By POLA1 Promoter in Proliferating and Directed Differentiation Resisting Human Autologous Pluripotent Induced Stem Cells Leads to their Death. J Stem Cell Res Ther S9: 005e. doi:10.4172/2157-7633.S9-005 [PubMed]

superparamagnetic or fluorescent antibodies targeting SSEA-4, SSEA3, TRA-1-81, and TRA-1-60 facilitated depletion of human pluripotent cells with the aid of magnetic (MACS) and fluorescent activated cell sorting (FACS) [13-18]. Fluorescent mouse monoclonal antibodies against SSEA-5 were used to remove the clones of pluripotent stem cells and reduce the number of teratomas [18]. Mouse monoclonal antibodies - mAb84 raised against podocalyxin-like protein-1 on undifferentiated human embryonic stem cells were capable for killing them [19]. Modified with toxins, mouse monoclonal antibodies against claudin-6, displayed on pluripotent stem cells, inflicted their death [20]. Combination of magnetic sorting and cytotoxic antibody followup led to selective removal of undifferentiated embryonic stem cells [21]. PluriSIn \#1, an inhibitor of stearoyl-co A desaturase and a key enzyme in oleic acid biosynthesis, was shown to be effective in killing of implanted human embryonic stem cells [22]. Transgenic expression of the recombinant thymidine kinase - cell suicide gene in human pluripotent stem cells made them lethally sensitive to Ganciclovir at doses lower than those toxic for adult cells [23-26].

The ultimate goal of our work is to develop a self-triggering feed-back mechanism, which eliminates the pluripotent stem cells that keep proliferating despite attempts of inducing their differentiation; thus which reliably prevents initiation of neoplasms. Unfortunately, none of the published strategies proposed a proliferation triggered cell suicide mechanism. Moreover, none of those strategies was protecting against risks of apoptosis processes' reversal, development of rescue mechanisms, or de novo proliferations' initiation, as it happens in cancer, which was the driving force for development of cancer suicide gene therapy [5,27-30]. Herein, we describe the novel strategy, which we have developed to safeguard stem cell therapy against iatrogenic cancerogenesis.

The specific aim was threefold: (1) to genetically engineer the DNA constructs for the human, recombinant DNASE1, DNASE1L3, DNASE2, DFFB controlled by POLA1 promoter; (2) to bioengineer anti-SSEA- 4 vectors delivering transgenes to undifferentiated bone marrow derived human induced pluripotent stem cells; (3) to cause death of the proliferating and non-differentiating stem cells by transgenic expression of the human recombinant DNases (hrDNases).

\section{Methods}

\section{Patients}

\section{Bone marrow}

\section{Cell culture}

All samples were obtained from patients undergoing marrow harvest for autologous transplantation in accordance with the Declaration of Helsinki with the Institutional Review Boards' Approval and with the Patients' Informed Consent. The cohort consisted of 3 men and 3 women, who agreed for using their bone marrow for research. All the surgical procedures were performed in the sterile conditions after induction of general anesthesia. Using heparinized, sterile needles, approximately $10 \mathrm{ml}$ volumes of bone marrow were aspirated from the iliac crests. No iatrogenic complications were ever reported. Cells from the aspirated marrow was either processed immediately, or expanded, or frozen.

For immediate analysis, the bone marrow aspirates were suspended in $20 \%$ Human Serum in Hank's Balanced Salt Solution $4^{\circ} \mathrm{C}$ on ice. These suspensions were very gently layered onto $1.077 \mathrm{~g} / \mathrm{mL}$ Ficoll (Pharmacia, Uppsala, Sweden) and spun $300 \mathrm{~g}$ for 25 minutes at $4^{\circ} \mathrm{C}$.
Bone marrow mononuclear cells (BMMCs) formed a band at the interface. They were aspirated from that band and the suspension/ centrifugation cycle repeated two more times.

For cell culture expansion, the cells were then resuspended in growth medium consisting of Iscove's modified Dulbecco's medium (IMDM) with $20 \%$ human serum, $4 \mathrm{mmol} / \mathrm{L}$ glutamine, $50 \mathrm{pg} / \mathrm{mL}$ penicillin and streptomycin (GIBCO, Grand Island, New York, USA), and $10 \mathrm{pmol} / \mathrm{L}$ hydrocortisone (Sigma, St Louis, MO). Growth was promoted by adding the following factors: $2 \mathrm{ng} / \mathrm{mL}$ rh interleukin-3 (R \& D Systems, Minneapolis, MN), $5 \mathrm{ng} / \mathrm{mL} \mathrm{hr}$ granulocyte-macrophage colonystimulating factor (Immunex, Seattle, WA), 0. $1 \mathrm{U} / \mathrm{mL}$ erythropoietin (Amgen, Thousand Oaks, CA, USA), and $10 \mathrm{ng} / \mathrm{mL}$ hr c-kit ligand (Immunex, Seattle, WA, USA). Large scale expansion of BMMCs was conducted according to conditions developed earlier for perfusion culture systems, while using bioreactors (New Brunswick Scientific, Hauppauge, NY, USA) [37]. The BMMCs were rinsed off cell culture media for further processing as described above.

For long term storage, the bone marrows aspirates were suspended in PBS supplemented with 5\% starch, 5\%DMSO, 30\% human serum for 15 min. on ice and cryoimmobilized in the programmable freezer (the freezer was designed and built based upon the NSF funds granted to Dr M. Malecki, the Principal Investigator) down to $-30^{\circ} \mathrm{C}$ at $1{ }^{\circ} \mathrm{C} / \mathrm{min}$, rapid cooling down to $-70^{\circ} \mathrm{C}$ at $30^{\circ} \mathrm{C} / \mathrm{min}$, and the final phase down to $-196^{\circ} \mathrm{C}$ at $3^{\circ} \mathrm{C} / \mathrm{min}$.

Bone marrow mononuclear cells were reprogrammed into human autologous pluripotent induced stem cells according to the detailed protocols already published earlier [33-37]. Batches of cells were depleted of apoptotic and necrotic cells by labeling with superparamagnetic synthetic antibodies against phosphatidylserine and double stranded DNA followed by magnetic activated cell sorting (MACS).

Bone marrow mononuclear cells were reprogrammed into human autologous pluripotent induced stem cells with the aid of the DNA plasmid constructs coding sequences of: NANOG, OCT4, SOX2, LIN28, KLF4, CMYC. These constructs had bioengineered reporting sequences to render them superparamagnetic or fluorescent $(\mathrm{Gd}, \mathrm{Eu})$; thus to facilitate determination of transfection efficacy. The hapiSCs cultures were clonally expanded to the desired quantities [16]. Resulting, reprogrammed pluripotent stem cells were directed to differentiate into endothelial cells. They were also transfected with the DNA plasmid constructs coding sequences of: DNASE1, DNASE1L3, DNASE2, DFFB. These constructs had bioengineered reporting sequences to render them superparamagnetic or fluorescent, but different than in those inducing pluripotency; thus to facilitate determination of transfection efficacy. They were transfected with the aid of the anti-SSEA-4 synthetic nano-antibody guided vectors as described below.

\section{Synthetic nano-antibodies against SSEA-4, TRA-1-60, PS, and dsDNA}

Synthetic nano-antibodies against SSEA-4 were bioengineered as described earlier and the sequences were published [13-16]. Briefly, fresh blood was received from the cancer patients according to the Declaration of Helsinki with the Institutional Review Board (IRB) approval and with the Informed Consent Forms (ICF) signed. White blood cells (WBC) were isolated using Ficoll-Hypaque technique. The $\mathrm{B}$ cells were isolated using genetically engineered antibodies targeting CD19 and CD20. The total mRNA was isolated using Trizol reagent (Molecular Research Center, Inc. Cincinnati, OH). The cDNA was generated using random hexamers (Intergrated DNA Technologies, 
Citation: Malecki M, Vanne CL, Alhambra D, Dodivenaka C, Nagel S, et al. (2013) Safeguarding Stem Cell-Based Regenerative Therapy against latrogenic Cancerogenesis: Transgenic Expression of DNASE1, DNASE1L3, DNASE2, DFFB Controlled By POLA1 Promoter in Proliferating and Directed Differentiation Resisting Human Autologous Pluripotent Induced Stem Cells Leads to their Death. J Stem Cell Res Ther S9: 005e. doi:10.4172/2157-7633.S9-005 [PubMed]

Page 3 of 9

Coralville, IA) and reverse transcriptase (Promega, Madison, WI) in reactions involving denaturing $\mathrm{RNA}$ at $70^{\circ} \mathrm{C}$ followed by reverse transcription carried at $42^{\circ} \mathrm{C}$ for $15 \mathrm{~min}$. The cDNA quality was tested by the polymerase chain reaction (PCR) of beta actin and GAPDH as reference genes with the commercially available primers (ABI, Foster City, CA). For amplification of variable fragments, the primers sets were designed using the Kabat's database. They were synthesized on the $380 \mathrm{~A}$ DNA Synthesizer (ABI, Foster City, CA). The variable fragments were amplified by polymerase chain reaction using the mix of the generated cDNA, the synthesized primers, dNTPs, and Taq DNA polymerase (Hoffmann-La Roche, Basel, Switzerland) using the Robocycler (Stratagene, San Diego, CA) or Mastercycler (Eppendorf, New York, $\mathrm{NY}$ ). The blunt ended amplicons were inserted into the $\mathrm{pM}$ construct containing the single EGFR transmembrane sequence imported from the GenBank Reference Sequence ID: NM_005228 in Public Domain (NCBI, Bethesda, MD). The DNA plasmid constructs also contained metal binding domains capable of chelating superparamagnetic and fluorescent metals. The constructs were electroporated and expressed in human myelomas. All the expressed clones were labeled in liquid phase with the free transgenic receptors, which were modified with fluorescent or superparamagnetic reporters. The clones expressing the heavy (HC) and light chains (VL) were selected on the fluorescent activated cell sorter (FACS) Calibur (Becton-Dickinson, Franklin Lakes, NJ) or magnetic activated cell sorter (MACS) (the sorter built based upon the grants from the NSF for Dr Malecki, the Principal Investigator). The new constructs were also expressed in human myelomas. The coding sequences were verified after total RNA extraction, reverse transcription, amplification, and sequencing of amplicons on the ABI 3130XL or Junior DNA Sequencer (ABI, Foster City, CA). The clones of the antibodies used to this study were encoded MR24 for the EGFRvIII and MS23 for the EGFR. For the first round of selections, the free, transgenic, soluble, extracellular domains of the receptors were generated as the baits. They were designed based upon the coding sequence for the human EGFRwt based upon the sequence imported as the NCBI Reference Sequence: AC006977.3 and for the human EGFRvIII carrying mutation deletion of the exons 2-7 as described and their sequences were published. The primers were designed using the Primer Express Primer Express (ABI, Foster City, $\mathrm{CA}$ ) and synthesized. After amplification and purification, the cDNA for the EGFR or EGFRvIII domains was transduced in myelomas followed by the gene expression products' purification on HPLC.

Synthetic nano-antibodies against dsDNA single chain variable fragments were bioengineered as described earlier and the sequences were published [33]. Briefly, the B cells were selected from the patients suffering from LE. They were sorted with MACS, after the DNA was modified with superparamagnetic antibodies. Alternatively, they were sorted by FACS, after the DNA was tagged with fluorescent reporters. RT PCR was performed on each cell carrying dsDNA targeting variable fragments. Coding sequences for the variable fragments were amplified and cloned within the plasmid vectors and expressed in human myelomas and B cells, as described and with all the sequences published $[33,37]$

\section{Bioengineering vectors for human recombinant DNASE1,} DNASE1L3, DNASE2, DFFB controlled by POLA1 promoter

\section{Studying effects of transgenic expression of DNases}

Tissue was obtained from cancer free margins during surgery of patients suffering from cancers of pancreas, liver, and ovary. Genomic DNA was isolated using Nucleic Acid Extractor Model 340A (ABI, Foster City, CA). Total mRNA was isolated using Trizol reagent (Molecu- lar Research Center, Inc. Cincinnati, OH). The cDNA was generated using random hexamers (Intergrated DNA Technologies, Coralville, IA) and reverse transcriptase (Promega, Madison, WI). The following coding sequences were imported from the NCBI and used to design the primers using PrimerBlast: homo sapiens deoxyribonuclease I (DNASE1): NCBI Reference Sequence: NC_000016.9; homo sapiens deoxyribonuclease 1L3 (DNASE1L3): NCBI Reference Sequence: NC_000003.11; homo sapiens deoxyribonuclease II (DNASE2): NCBI Reference Sequence: NC_000019.9; homo sapiens DNA fragmentation factor B (DFFB): NCBI Reference Sequence: NC_000001.10. The primers were synthesized on the 380A DNA Synthesizer (ABI, Foster City, CA) and the sequences amplified on the Robocycler (Stratagene, San Diego, CA), Mastercycler (Eppendorf, Hamburg, Germany), or 7500, 7900 HT qPCR systems (ABI, Foster City, CA). The following coding sequences were imported from the NCBI and synthesized on the DNA synthesizer: homo sapiens promoter for POLA1: NCBI Reference Sequence: HF584050.1 (GCGCCTGTCTCGGCCCCCGCGCCAGTTTTGGGCTGGTTGGCGCGGAATCGGGAGATTCGG) (Prom_POLA1), homo sapiens nuclear localization signal for nucleoplasmin (NPM1) (NLS_NPM1), and short unique tagging sequence. The coding sequences for each of the DNases were joined by overlap extension with those for the Prom-POLA1 and NLS-NPM. As fluorescent reporters, the following coding sequences for fluorescent proteins within the plasmids were according to: GFP as in GenBank Accession M62653.1 (the gift from Dr D. Prasher) and its BFP, CFP, and YFP mutations (the gift from Dr R. Tsien) [38-41]. Alternatively, as superparamagnetic and elemental reporters, the sequences harboring for $\mathrm{Gd}, \mathrm{Eu}, \mathrm{Tb}, \mathrm{Ni}$, Co chelators were synthesized [33]. Those sequences were inserted to code carboxyl termini of the expressed fusion proteins. These four DNases were assembled into the transfection vectors, which were bioengineered as described [27]. Briefly, the synthetic antibodies against DNA were carrying biotin tag at the carboxyl termini. After binding the DNA constructs for the DNases, they were docked into the biotin binding site of the recombinant avidin one at a time to create the DNA non-viral vectors. These vectors were guided by synthetic biotags into the cells as described [37]. These vectors carrying plasmids of the same sizes, but with reversed direction coding sequence for DNases or without NLS were delivered as the controls. Since, the biotags carried permanent fluorescent, radionuclide, or superparamagnetic reporters, efficacy of targeted delivery was easy to quantify with MPFS, EDXS, GRS, or NMRS.

Effects of transgenes' expression were determined by MPFS of living cells' chromatin and electrophoresis of nuclei's lysates. Surfaces of cryo-immobilized cells were studied by FESEM. Architecture of nuclear chromatin was revealed by EFTEM. Apoptotic and necrotic cells were quantified after labeling with synthetic antibody based biotags against dsDNA and against PS. These biotags were rendered fluorescent or superparamagnetic, so that quantification of dead or apoptotic cells was pursued with FCM, XRFS, or NMRS.

\section{Directing vasculogenesis of human pluripotent stem cells}

Upon completion of the recruitment of the bone marrow stem cells to the cardiac tissues, the solution, flowing through the chambers, was Vascular Cell Basal Medium (ATCC, Arlington, VA, USA) supplemented with recombinant human Vascular Endothelial Growth Factor $50 \mathrm{ng} / \mathrm{mL}$, recombinant human Epidermal Growth Factor 5 ng/mL, recombinant human Basic Fibroblast Growth Factor 5 ng/ $\mathrm{mL}$, recombinant human Insulin-like Growth Factor $15 \mathrm{ng} / \mathrm{mL}$, Angiopoietin-1 $20 \mathrm{ng} / \mathrm{mL}$, L-glutamine $10 \mathrm{mM}$, heparin sulfate 0.75 Units $/ \mathrm{mL}$, hydrocortisone hemisuccinate $1 \mu \mathrm{g} / \mathrm{mL}$, ascorbic acid 50 
Citation: Malecki M, Vanne CL, Alhambra D, Dodivenaka C, Nagel S, et al. (2013) Safeguarding Stem Cell-Based Regenerative Therapy against latrogenic Cancerogenesis: Transgenic Expression of DNASE1, DNASE1L3, DNASE2, DFFB Controlled By POLA1 Promoter in Proliferating and Directed Differentiation Resisting Human Autologous Pluripotent Induced Stem Cells Leads to their Death. J Stem Cell Res Ther S9: 005e. doi:10.4172/2157-7633.S9-005 [PubMed]

$\mu \mathrm{g} / \mathrm{mL}$. At various time intervals, vasculogenesis of the retained stem cells was quantified. Human Normal Artery Endothelial Cells (ATCC, Arlington, VA, USA) served as the positive controls. The tissues were homogenized, followed by extraction of mRNA, quantitative reverse transcription and polymerase reaction amplification of the transcripts for claudin, ZO-1, VE cadherinm and occludin. The cells were labeled with the antibodies for to highlight in multiphoton fluorescence microscope the gene expression products forming tight and adherens junctions.

\section{Flow cytometry (FCM). Fluorescently activated cell sorting} (FACS). Multiphoton Fluorescence Spectroscopy (MPFS)

For flow cytometry (FCM), fluorescent activated cell sorting (FACS) and magnetic activated cell sorting (MACS) were thoroughly prepared as single cell suspensions by short treatment with the PIPES buffered DNase, RNase, trypsin, collagenase, or dispase II ( 0.5 units $/ \mathrm{mg})$. These preparations included cell clusters and embryoid bodies. Moreover, apoptotic cells were removed with the Fvs anti-PS and dead cells with the Fvs anti-DNA. The enriched populations of the cells labeled with the fluorescent Fvs targeting TRA-1-60, TRA-1-81, SSEA-3, SSEA-4 were measured with the Calibur, Vantage SE, or Aria (Becton-Dickinson, Franklin Lakes, NJ, USA) or the FC500 (Beckman-Coulter, Brea, CA, USA). The fluorescently labeled cells were imaged with the Axiovert (Zeiss, Oberkochen, D, EU) equipped with the Enterprise argon ion (457 nm, $488 \mathrm{~nm}, 529 \mathrm{~nm}$ lines) and ultraviolet (UV) (364 nm line) lasers; Odyssey XL digital video-rate confocal laser scanning imaging system operated up to 240 frames/s under control of Intervision software (Noran, Madison, WI, USA), and the Diaphot (Nikon, Tokyo, Japan) equipped with the Microlase diode-pumped Nd:YLF solid state laser (1048 $\mathrm{nm}$ line), the pulse compressor with the pulses' rate $300 \mathrm{fs}$ at $120 \mathrm{MHz}$ and the MRC600 scanning system under control of Comos software (the multi-photon fluorescence station built based upon the NIH funds - PI: Dr J. White). Deconvolution of images was done on the Indy workstation (Silicon Graphics, Fremont, CA, USA).

\section{Nuclear Magnetic Resonance Spectroscopy (NMRS). Magnetic Activated Cell Sorting (MACS)}

The cells were labeled for positive selection with the superparamagnetic Fvs targeting TRA-1-60 and SSEA-4, and for the negative selection targeting CD45, CD34, dsDNA, and PS, while suspended in the physiological buffer supplemented with serum and glucose. The small aliquots were dispensed into the magnetism-free NMR tubes (Shigemi, Tokyo, Japan). The relaxation times T1 were measured in resonance to the applied FLAIR pulse sequences on the NMR spectrometers: DMX 400 WB or AVANCE II NMR (Bruker, Billerica, MA) or the Signa clinical scanners (GE, Milwaukee, WI, USA). The superparamagnetic Fvs were also used to isolate the labeled cells from the solution using the magnetic sorter to reach above $99.5 \%$ of purity (the sorter designed and built based upon the NSF funds - PI: Dr M. Malecki).

Electron Energy Loss Spectroscopy (EELS). Energy Dispersive X-Ray Spectroscopy (EDXS). X-ray Reflection Fluorescence Spectroscopy (XRFS)

The samples, which were cryo-immobilized, presented the lifelike supramolecular organization. Molecular imaging was pursued as described. The field emission, scanning transmission, electron microscope FESTEM HB501 (Vacuum Generators, Kirkland, WA, USA) was equipped with the energy dispersive $\mathrm{x}$-ray spectrometer (EDXS) (Noran, Middleton, WI, USA) and post-column electron energy loss spectrometer (EELS) (Gatan, Pleasanton, CA). The cryo- energy filtering transmission electron microscope 912 Omega was equipped with the in-column, electron energy loss spectrometer (EELS) (Zeiss, Oberkochen, D, EU). The cryo-energy filtering transmission electron microscopes 410 and 430 Phillips were equipped with the postcolumn, electron energy loss spectrometers (EELS) (Noran, Middleton, WI, USA). The field emission, scanning electron microscope SEM1530 (Zeiss, Oberkochen, D, EU) was equipped with the energy dispersive $\mathrm{x}$-ray spectrometer (EDXS) (Noran, Middleton, WI, USA). The field emission, scanning electron microscope 3400 was equipped with the energy dispersive x-ray spectrometer (EDXS) (Hitachi, Tokyo, Japan). The images and spectra were acquired using the ccd camera operating under the image acquisition and processing software (SIS, Herzogenrath, D, EU or Emispec Systems, Tempe, AZ, USA). In the XRFS study, the ICP standard of $1000 \mathrm{mg} / \mathrm{l}$ of mono-element Gallium (CPI International, Denver, CO, USA) was added to 500 microL of each sample to the final concentration of $10 \mathrm{mg} / \mathrm{l}$. The data were generated from the S2 Picofox TXRF spectrometer equipped with a molybdenum (Mo) X-ray target and the Peltier cooled Xflash Silicon Drift Detector (Bruker AXS, Fitchburg, WI, USA). Scan times ranged up to 1000 seconds. The automatic sample changer, which can hold up to 25 samples, was also used along with the SPECTRA 7 software for the instrument control, data collection, and analysis (Bruker AXS, Fitchburg, WI, USA).

\section{Immunoblotting (IB)}

The cells and tissues were frozen in liquid nitrogen, crushed, and thawed or/and disintegrated with the ultrasonicator (Branson Ultrasonic, Danbury, CT, USA) within the sample buffers for native protein analysis. They were stored in liquid nitrogen or electrophoresed in the native buffer (Invitrogen, Carlsbad, CA, USA). They were vacuum or electro-transferred onto the PVDF membranes (Amersham, Buckinghamshire, UK, EU). The membranes carrying the transferred proteins were soaked within human serum and labeled with the Fvs. The samples of purified cardiac muscle myosin, actin, $a$-actinin, titin served as the controls. The commercially available monoclonal antibodies against myosin, actin, $\alpha$-actinin, titin, SSEA3, SSEA4, TRA160, TRA181, CD34, and CD45 served as the controls. The images of the blots were acquired and quantified with Fluoroimager (Molecular Dynamics, Sunnyvale, CA, USA) or Storm 840 (Amersham, Buckinghamshire, UK, EU). The levels of the gene expression products were also calculated, as the ratio between the protein concentration in the examined patient's cells and the controls.

\section{Quantitative Reverse Transcription and Polymerase Chain Reaction (qRTPCR)}

Total RNA was isolated with TRIzol (MRC, Cincinnati, OH, USA), In addition to the patients' cardiac tissues, the fibroblasts, peripheral blood cells, and bone marrow cells were processed. The cultured fibroblasts (IMR90), human embryonic stem cells (H1, H9), and blood from the healthy volunteers served as the controls. For all, RNA served as the template to generate cDNA through reverse transcription using random hexamers and reverse transcriptase (ABI, Foster City, CA, USA). The primers' sequences and cycling settings were modified from those given. The transcripts for GAPDH and actin served as the internal controls (ABI, Foster City, CA, USA). They were synthesized on the 380A DNA Synthesizer (ABI, Foster City, CA, USA). The PCR reactions were carried using the mix of the CDNA, the synthesized primers, dNTPs, and Taq DNA polymerase (Hoffmann-La Roche, Basel, H) on the Robocycler (Stratagene, San Diego, CA, USA), Mastercycler (Eppendorf, Hamburg, D, EU), or 7500, 7900 systems 
Citation: Malecki M, Vanne CL, Alhambra D, Dodivenaka C, Nagel S, et al. (2013) Safeguarding Stem Cell-Based Regenerative Therapy against latrogenic Cancerogenesis: Transgenic Expression of DNASE1, DNASE1L3, DNASE2, DFFB Controlled By POLA1 Promoter in Proliferating and Directed Differentiation Resisting Human Autologous Pluripotent Induced Stem Cells Leads to their Death. J Stem Cell Res Ther S9: 005e. doi:10.4172/2157-7633.S9-005 [PubMed]

Page 5 of 9

(ABI, Foster City, CA, USA). The images of the gels were acquired and quantified with Fluoroimager (Molecular Dynamics, Sunnyvale, CA, USA) or Storm 840 (Amersham, Buckinghamshire, UK, EU). The levels of the transcripts were all normalized against GAPDH or actin. Thereafter, they were calculated as the ratios between the transcripts' concentration in the examined patient's cells versus the cells from the healthy control tissues and cultures.

\section{Statistical analysis}

All the measurements were run in triplicates for each sample from six patients (three women and three men). The numbers were analyzed and displayed using GraphPad software (GraphPad Software, Inc, La Jolla, CA). Data were presented as mean \pm standard error of the mean (SEM). Statistical significance was calculated by t-test for two groups.

\section{Results}

We have determined the effects of transgenic expression of the genes for the human, recombinant DNases (hrDNases) on human autologous pluripotent induced stem cells, while discriminating these effects between proliferating and differentiating stem cells. As the positive control, we have determined the effects of transgenic DNases' expression on the human embryonic stem cells. As the negative control, we have determined the effects of transgenic DNases' expression on the human artery endothelial cells and the human induced endothelial cells. We have compared the results of experiments on the human autologous pluripotent induced and embryonic stem cells transfected with the vectors carrying recombinant genes for DNases with the results of the same experiments conducted on cells transfected with the vectors carrying the ORF in reversed direction and those not exposed to transfecting vectors at all.

We have pursued research with several integrated strategies: (1) directing differentiation of induced pluripotent stem cells into endothelial cells, while imaging by multiphoton fluorescence of structural proteins of tight and adherens junctions as fusions with green fluorescence proteins and their mutants; (2) analyzing the cells' surface topography in differentiating versus proliferating cells by field emission scanning electron microscopy (FESEM); (2) watching externalization of phosphatidylserine (PS) in stem cells undergoing apoptosis; presented are representative to (3) studying architecture of genomic DNA (gDNA) in living cells; (4) evaluating integrity of genomic DNA (gDNA) isolated from proliferating cells, which were transfected with the vectors for the hrDNases; (5) revealing ultrafine architecture of chromatin with field emission energy filtering transmission electron microscopy (FEEFTEM); (6) quantifying the deadly effects of the transgenic DNases expression by labeling with molecular death tags for nuclear magnetic resonance spectroscopy (NMRS); (7) quantifying these deadly effects by energy dispersive $\mathrm{x}$-ray spectroscopy (EDXS).

Directed differentiation of the human autologous pluripotent stem cells induced from bone marrow mononuclear cells resulted in formation of endothelial cells (Figure 1). Accuracy and selectivity of the vectors to deliver the transgenes for the hrDNases into the undifferentiated pluripotent stem cells did depend on high specificity of the guiding synthetic antibodies as validated earlier [13,14]. Differentiation was verified by recording assembly of tight junctions highlighted by transgenic gene expression product - blue mutant of green fluorescent protein fusion with zona occludens 1 and by labeling with synthetic antibody against extracellular domain of claudin. It was also verified by recording assembly of adherens junctions highlighted by transgenic gene expression product - yellow mutant of green fluorescent protein fusion with catenin and by labeling with synthetic antibody against extracellular domain of vascular endothelial cadherin. These cells did not display any of the markers of the undifferentiated proliferating stem cells including SSEA-4, SSEA-3, TRA-1-60, and TRA-1-81. These cells were not penetrated by the anti-dsDNA antibodies or propidium iodide. Each of the assays was conducted in triplicates. The data presented are representative to all samples tested.

Human autologous pluripotent induced stem cells and human embryonic stem cells were stimulated to differentiate with VEGF and BMP. They were also transfected with the transgenes coding DNASE1, DNASE1L3, DNASE2, DFFB controlled by POLA promoter. We resorted to ultrastructural imaging by field emission scanning electron microscopy (FESEM) of surface topographies on cryo-immobilized and freeze-dried cells to detect the earliest signs of death (Figure 2). Human autologous pluripotent stem cells, which were induced from bone marrow mononuclear cells, were having regular round geometry and surfaces projected as multiple, fine filopodia. Their batches were depleted of necrotic and apoptotic cells with superparamagnetic antibodies against PS and dsDNA. As the positive control, we have determined the effects of transgenic DNases' expression on the human embryonic stem cells. As the negative control, we have determined the effects of transgenic DNases' expression on the human artery endothelial cells and the human induced endothelial cells. All the assays were conducted in triplicates and those shown are representative to all studied. The proliferating, undifferentiated human autologous pluripotent induced stem cells, which were not transfected with the hrDNases' vectors, were having classical geometry joined by an isthmus. In the batches of the human autologous pluripotent induced stem cells, which were transfected with the vectors for the hrDNases, there were cells, which showed first signs of apoptosis-like changes cell surface blebs, already within an hour. Magnitude of these changes and numbers of the affected cells rapidly increased over time to reach the point, when all undifferentiated stem cells were affected.

Molecular nature of blebs forming on surfaces of the cells transfected with the vectors for the DNases was probed by labeling with antibodies to phosphatidylserine (PS) on living cells (Figure 3). Three rounds of assays were performed. The images are representative to all acquired. Synthetic nano-antibodies, which guided the vectors, demonstrated

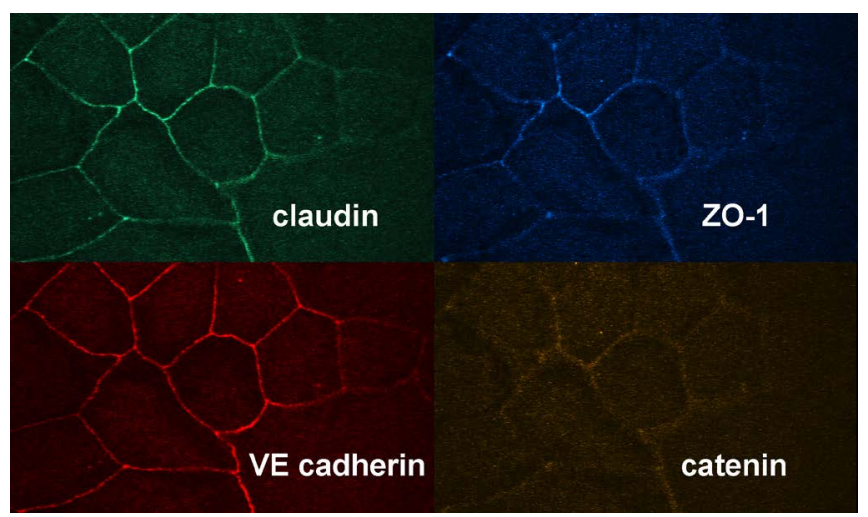

Figure 1: Directed differentiation of the human bone marrow derived autologous pluripotent induced stem cells into endothelial cells. Gene expression products for and assembling of tight junctions: claudin 5 and zona occludens 1 (ZO-1) and adherens junctions: vascular endothelium cadherin and catenin prove efficacy of directed differentiation. Claudin and VE cadherin were highlighted by fluorescent red and cyan synthetic nano-antibodies. ZO-1 and catenin were highlighted by transgenic expression of their fusions with blue and yellow mutants of fluorescent proteins. 
Citation: Malecki M, Vanne CL, Alhambra D, Dodivenaka C, Nagel S, et al. (2013) Safeguarding Stem Cell-Based Regenerative Therapy against latrogenic Cancerogenesis: Transgenic Expression of DNASE1, DNASE1L3, DNASE2, DFFB Controlled By POLA1 Promoter in Proliferating and Directed Differentiation Resisting Human Autologous Pluripotent Induced Stem Cells Leads to their Death. J Stem Cell Res Ther S9: 005e. doi:10.4172/2157-7633.S9-005 [PubMed]

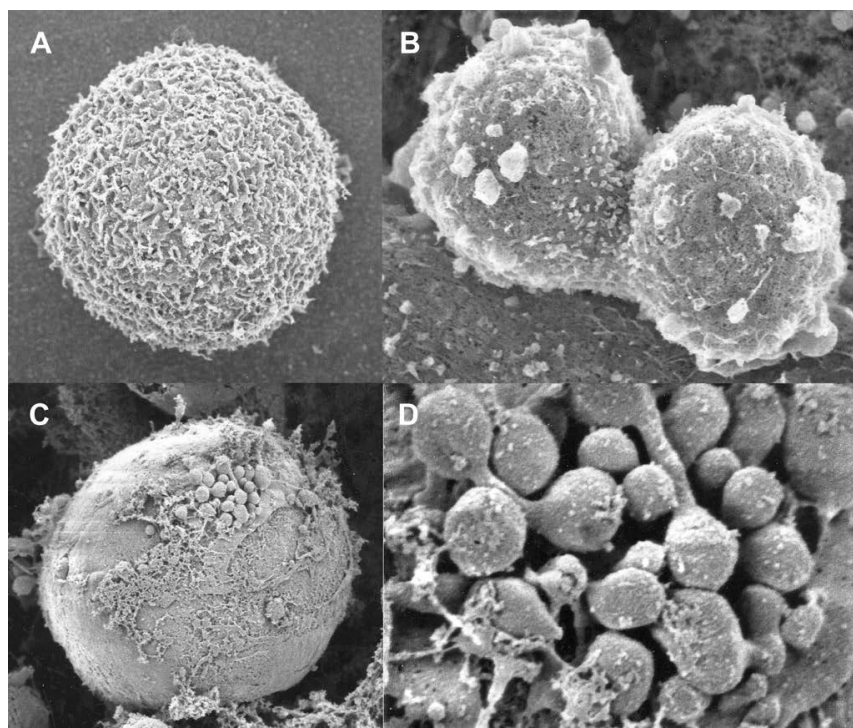

Figure 2: Human bone marrow and pluripotent induced stem cells directed to die. Human bone marrow mononuclear cells were induced into human autologous pluripotent stem cells, which displayed cell surface biomarker SSEA-4, and selected with superparamagnetic synthetic nanoantibodies by MACS. After rapid cryoimmobilization, the cells were imaged with field emission scanning electron microscopy. The cells have round geometry and classical cell surface architecture (A). They vigorously proliferate, what is reflected in their geometry and cell surface architecture (B). The cells were transfected with the SSEA-4 antibody guided vectors coding DNASE1, DNASE1L3, DNASE2, and DFFB controlled by POLA promoter. Early signs of these transgenes' expression, concurrent with $\mathrm{G} 1 / \mathrm{S}$ transition, were blebs altering cell surface architecture of the human autologous induced pluripotent stem cells (C). Some of these membrane blebs were developing advanced porosity - open routes for molecular entries (D).

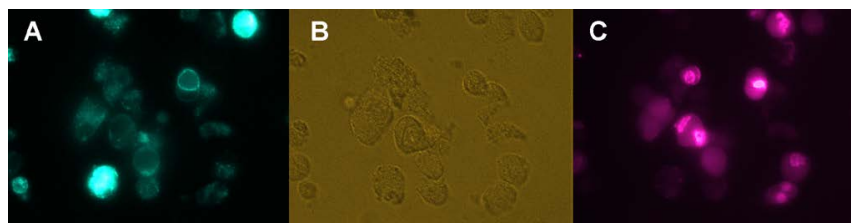

Figure 3: Molecular symptoms of human recombinant DNases' directed suicide of proliferating stem cells. Human autologous pluripotent induced stem cells were transfected with the SSEA-4 antibody guided vectors carrying coding sequences for DNASE1, DNASE1L3, DNASE2, and DFFB controlled by POLA promoter. Early signs of these transgenes' expression, concurrent with $\mathrm{G} 1 / \mathrm{S}$ transition, were manifested by externalization of phosphatidylserine (A) and membrane blebs corresponding to those shown in the figure 2 . Zernicke's phase contrast served as the reference (B). Transgenic expression of the human recombinant DNases resulted in complete collapse of chromatin architecture (C).

high specificity and exquisite sensitivity as determined previously [13-14]. Within the time frames identical to those recorded during detection of cell surface blebbing, externalization of PS was noticed. Concurrently with those alterations, nuclear chromatin was suffering structural degradation, which was leading to its complete collapse. Those changes were entirely absent in the differentiated stem and endothelial cells serving as the controls, as well as in the cells which were transfected with the vectors carrying ORF in the reversed orientation.

To further refine observations on the living human autologous pluripotent induced and embryonic stem cells, integrity of genomic DNA was tested by isolation and electrophoresis without prior ultrasonic fragmentation or restriction enzymes cutting (Figure 4).
Genomic DNA was also isolated from the cells, which were exposed to the vectors carrying the ORF in the reversed orientation and from those, which were not transfected at all. Assays were conducted in triplicates and representative results presented. Genomic DNA from proliferating stem cells, which were transfected with the vectors for DNase1, DNase1L3, DNase2, DFFB was completely degraded. It appeared as comet-like smudges along the lanes. Genomic DNA from the cells, which were transfected with the vectors carrying reversed orientation ORF or which were not transfected remained mostly in loading wells.

The ultimate goal of the work was to direct proliferating stem cells to their death with no options for reversal of any part of the process. That could happen through the complete degradation of nuclear chromatin architecture and degradation of genomic DNA by expression of the trangenes for the hrDNases and intranuclear targeting of the transgene expression products. To study this phenomenon, we resorted to ultrastructural imaging of chromatin ultrafine architecture in situ by field emission energy filtering transmission electron microscopy (FEEFTEM) (Figure 5). At least fifty cells were imaged from each of the samples. Presented images illustrate the features unique to all studied. In the batches of proliferating cells expressing the hrDNases, chromatin was suffering from various degrees of degradation. Ultrafine architecture of the cells, which were transfected with the vectors carrying ORF in the reversed orientation or which were not transfected, remained unaffected. The observed ultrastructure was in perfect accord with the data acquired in the studies on living cells and extracted genomic DNA (Figures 1 and [33]).

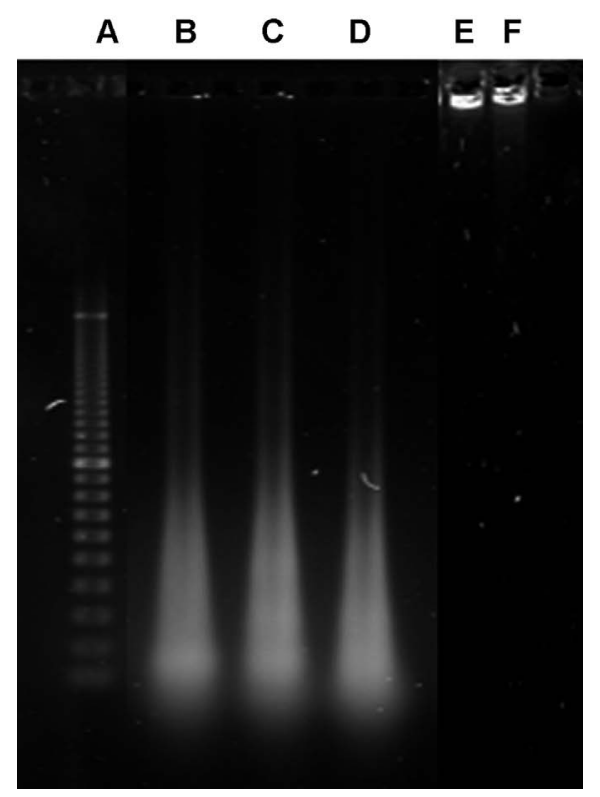

Figure 4: Degradation of genomic DNA as the result of transgenic expression of the human recombinant DNases. Genomic DNA was extracted from the human autologous pluripotent induced stem cells (B) human embryonic stem cells $(C)$, and embryonal carcinoma of the ovary (D), were transfected with the anti-SSEA-4 antibody guided vectors carrying coding sequences for DNASE1, DNASE1L3, DNASE2, and DFFB controlled by POLA1 promoter. The hapiSCs transfected with the vectors carrying ORF in reversed orientation $(E)$, and without the vector $(F)$ served as the controls. The 100bp ladder was the reference. Genomic DNA in the transduced cells is completely degradaded (B-D). Genomic DNA of the non-transfected and transfected with the vectors carrying reversed ORF was intact and as such remained in the loading wells $(E-F)$. 
Citation: Malecki M, Vanne CL, Alhambra D, Dodivenaka C, Nagel S, et al. (2013) Safeguarding Stem Cell-Based Regenerative Therapy against latrogenic Cancerogenesis: Transgenic Expression of DNASE1, DNASE1L3, DNASE2, DFFB Controlled By POLA1 Promoter in Proliferating and Directed Differentiation Resisting Human Autologous Pluripotent Induced Stem Cells Leads to their Death. J Stem Cell Res Ther S9: 005e. doi:10.4172/2157-7633.S9-005 [PubMed]

Page 7 of 9

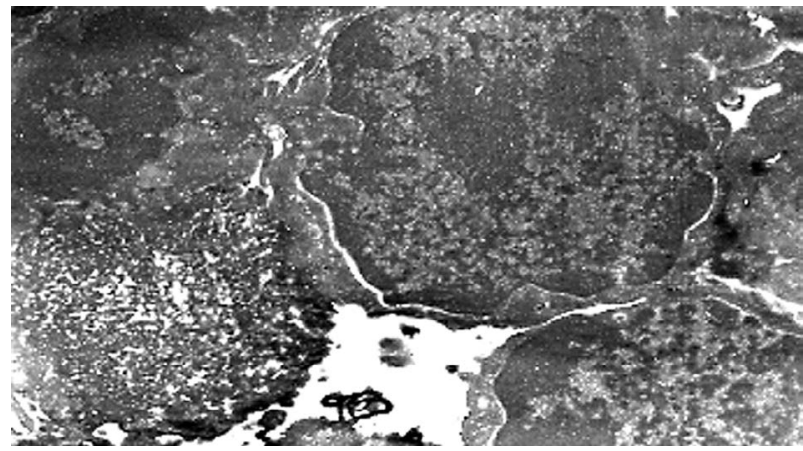

Figure 5: Collapse of the ultrafine architecture of chromatin as the result of transgenic expression of the hrDNases. Human autologous pluripotent induced stem cells were directed to differentiate, while transfected with the SSEA-4 antibody guided vectors carrying coding sequences for DNASE1, DNASE1L3, DNASE2, and DFFB controlled by POLA1 promoter. The vectors delivered the transgenes for the hrDNases'only into the SSEA-4 displaying cells. Expression of these hrDNases occurred only in undifferentiated and proliferating stem cells, but did not occur in the differentiated cells. After rapid cryoimmobilization, the cells were imaged with field emission energy filtering transmission electron microscopy.

Efficacy for inducing the proliferating cells' death was quantified with nuclear magnetic resonance spectroscopy (NMRS) (Figure 6). Specificity of the acquired data was confirmed by energy dispersive $\mathrm{x}$-ray spectroscopy (EDXS). These were all non-destructive analytical approaches, which ensured retention of the viable cells' for continued analysis, i.e., cell counts were followed by sorting of the previously studied living cells. Each assay was repeated three times and the results were averaged. Progression of apoptosis and/or secondary necrosis, due to transgenic expression of the hrDNases, was quantified by labeling of the cells with superparamagnetic antibodies against dsDNA and measuring the samples' relaxivities with NMRS. That followed by separation of the labeled cells with MACS. A large fraction of the proliferating cells, which were transfected with the vectors for the hrDNases, were dead within the first hour since the transfection. Almost all of these cells were confirmed dead within eight hours. The cells, which were transfected with the vectors carrying ORF in the reversed orientation or which were not transfected, retained high viability. Efficacy for inducing cells' death was also quantified by scintillation counts in energy dispersive $\mathrm{x}$-ray spectroscopy (EDXS) from the proliferating human autologous pluripotent induced and embryonic stem cells versus endothelial cells from directed differentiation endeavors and bioreactor cultures, which were all labeled with the elemental tagged synthetic antibodies. The scintillation data confirmed those from relaxivities' studies. Transgenic expression of the hrDNases was very effective in inducing death of proliferating human autologous pluripotent induced and embryonic stem cells, but had no effects upon differentiated endothelial cells.

\section{Discussion}

Herein, we describe attaining the proof-of-concept for the strategy, whereby transgenic expression of the genetically engineered human recombinant DNases in proliferating and directed differentiation resisting, but not in differentiating, stem cells leads to their death. The following main factors contributed to successful attaining of the project's aims: (1) specificity of the vectors; (2) cell cycle specific transgenes' expression; (3) intranuclear targeting of the transgenic hrDNases; (4) full spectrum of the hrDNases' optima; (5) irreversibility of the deadly processes.
1. Viral and stem cell vectors are most often used to deliver therapeutic transgenes. Specificity of their therapeutic delivery is contingent of their tropisms. However, none of them is uniquely specific to proliferating stem cells. Therefore, our bioengineering of the vectors, which target undifferentiated stem cells only, ensures exquisite specificity for reagents delivery. If it goes to clinical trials, it should be translated into absence of side effects caused by delivering upon these reagents into healthy cells.

2. Expression of POLA1 is strictly related to the phases of the cell cycle during their proliferation. It rapidly increases only in cells' preparation for G1/S transition. Therefore, only proliferating cells, having passed all the checkpoints and preparing DNA synthesis, experience increased levels of the DNA polymerases. This feature is incorporated into our safeguarding strategy by putting the gene constructs for the human DNases under the control of POLA1. Therefore, in undifferentiated and proliferating cells, the signal to proliferate is equivalent of the signal to die.

3. The essential element of our strategy is genetic engineering of the sequences coding for nuclear localization signal into the DNA constructs coding for the recombinant DNases. Therefore, the gene expression products - human recombinant DNases are guided into the cell nuclei ready to degrade the genomic DNA immediately upon completed translation.

4. DNases have their operational optima depending on the location of their tasks. These optima differ, e.g., for pancreatic or lysosomal DNases. Environments in cellular compartments of dying cells change dramatically. Therefore, adjusting the recombinant DNases operational optima and covering the entire spectra of operations by the four main DNases, which we used, created most efficient cocktail capable for permanent degradation of genomic DNA.

5. Cancer stem cells were recognized as becoming capable for expelling therapeutics or repairing damaged genomic DNA. Both mechanisms may help them to avoid their eradication. However, both these mechanisms require genomic DNA to stay intact. Our approach relies upon complete degradation of genomic DNA in proliferating stem cells, but not in differentiated cells. Therefore, our strategy ensures permanent eradication of the proliferating stem cells.

The main problems of this strategy are: (1) interception of the vectors by reticulo-endothelial system (RES); (2) immunogenicity of the vectors.

1. If applied in vivo, the vector of this size may become intercepted by the patients' reticulo-endothelial system. This may reduce its efficacy. If it happens during the in vivo trials, the biostealth molecules including polyethylene-glycol may have to be applied.

2. The components of the vectors may become immunogenic after multiple applications. Therefore, reaching high therapeutic efficacy already during first applications may be beneficial for avoiding this problem. As with immuno-therapies immunosuppression may also be applied in clinical trials. Moreover, using neutralizing antibodies, which we described earlier, may help to resolve this problem in clinical trials.

Mechanisms of the cells' death, which is induced by transgenic expression of the hrDNases, are complex due to the composite nature 
Citation: Malecki M, Vanne CL, Alhambra D, Dodivenaka C, Nagel S, et al. (2013) Safeguarding Stem Cell-Based Regenerative Therapy against latrogenic Cancerogenesis: Transgenic Expression of DNASE1, DNASE1L3, DNASE2, DFFB Controlled By POLA1 Promoter in Proliferating and Directed Differentiation Resisting Human Autologous Pluripotent Induced Stem Cells Leads to their Death. J Stem Cell Res Ther S9: 005e. doi:10.4172/2157-7633.S9-005 [PubMed]

hrDNAses' induced death of hapiSCs contingent upon DNA constructs

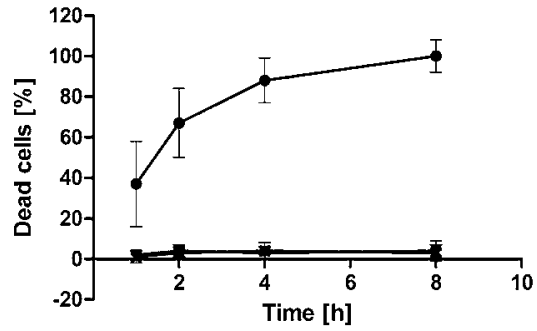

hrDNAses' induced death of hapiSCs and heSCs vs HUVEC and i-EC
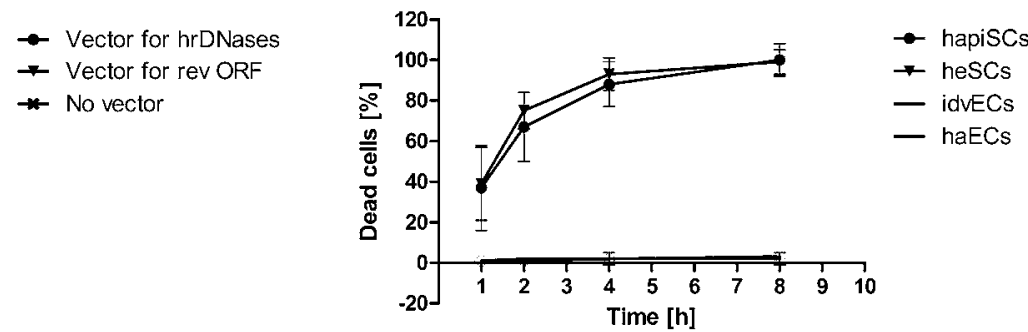

Figure 6: Statistical evaluation of directed suicide in proliferating stem cell. A. The human autologous induced pluripotent stem cells treated with the vectors for the hrDNases (Vector for hrDNases), with the vectors carrying the ORF in the reversed orientation (Vector for rev ORF), or with physiological buffer containing no vectors (No vector). At different time intervals, the cells were labeled with the superparamagnetic synthetic nano-antibodies targeting dsDNA. The results were quantified by measuring changes relaxivities detected in NMRS. First signs of directed stem cells' death were already detected within the first hour since transfection. B. The human autologous induced pluripotent stem cells (hapiSCs), human embryonic stem cells (heSCs), endothelial cells generated by induced directed vasculogenesis (idvECs), and human artery endothelial cells (haECs) were treated the vectors for the hrDNases. At different time intervals, the cells were labeled with the element tagged antidsDNA antibodies and the scintillation were counted in EDXS. proliferating human autologous induced pluripotent stem cells and human embryonic stem cells were induced to die. Differentiated endothelial cells remained unaffected.

of the therapeutic cocktail. Altogether, they all contribute to the complete degradation of genomic DNA. Mechanisms of apoptosis or necrosis, caused by external factors, involve activation of signaling cascades leading to the cells' death. Symptoms of consecutive stages can be distinguished. Early stages of necrosis include swollen cell volume, dilation of organelles, ruptured plasma membrane, and spill of intracellular contents. Early stages of apoptosis are characterized by cell externalization of phosphatidylserine, membrane blebs, which are first signs of cell membranes loosing integrity and becoming permeable, collapse of the chromatin architecture. The grand finale of apoptosis is activation of caspases and DNases, which ultimately, if not reversed, lead to degradation of genomic DNA. Those may be followed by secondary necrosis. The mechanisms of "death from inside" described herein, which is induced by complete degradation of the genomic DNA in the proliferating, but not in differentiating, stem cells due to transgenic expression of the four hrDNases, involve overlapping, interference, and integration of all those processes.

\section{Conclusion}

Herein, we describe attaining the proof-of-concept for the strategy, whereby transgenic expression of the genetically engineered human recombinant DNases in proliferating and directed differentiation resisting stem cells leads to their death. This novel strategy reduces the risk of iatrogenic cancerogenesis in stem cell therapy.

\section{Acknowledgment}

We thank the patients for the consent. We gratefully acknowledge providing primers, hexamers, monoclonal antibodies, tissues, and cells, as well as sharing valuable comments by Dr.M.Anderson, Dr.P.Andrews, Dr.J.Antosiewicz, Dr.A.Bradbury, Dr.T.Kunicki, Dr.J.Markley, Dr.J.Pietruszkiewicz, Dr.J.V.Small, Dr.S.Sidhu, Dr.W.Szybalski, Dr.J.Thomson.

\section{Conflict of Interest Statement}

None to declare. Marek Malecki MD PhD owns the intellectual property for the transgenes' constructs, their transcripts and products used for this work, which are all protected by USPTO and WIPO

Sources of Funding for the Work: This work was supported by the funds from the National Science Foundation [grant numbers: 9420056, 9522771, 9902020, and 0094016]; from the National Institutes of Health [grant numbers: P41 RR000570 and P41 RR002301]; and from the Phoenix Biomolecular Engineering Foundation [grant number: 2006070101] to Marek Malecki MD PhD, the Principal Investigator. Administrators of the funding institutions and managers of the facilities had no influence on the project design and presented data.

\section{References}

1. Takahashi K, Yamanaka S (2013) Induced pluripotent stem cells in medicine and biology. Development 140: 2457-2461 [PubMed].

2. Scadden D, Srivastava A (2012) Advancing stem cell biology toward stem cell therapeutics. Cell Stem Cell 10: 149-150 [PubMed].

3. Li M, Chen M, Han W, Fu X (2010) How far are induced pluripotent stem cells from the clinic? Ageing Res Rev 9: 257-264 [PubMed].

4. Hyun I (2010) The bioethics of stem cell research and therapy. J Clin Invest 120: 71-75 [PubMed].

5. Malecki M (2012) Frontiers in suicide gene therapy of cancer. J Genet Syndr Gene Ther 4: 1-41 [PubMed].

6. Knoepfler PS (2012) Key anticipated regulatory issues for clinical use of human induced pluripotent stem cells. Regen Med 7: 713-720 [PubMed].

7. Goldring CE, Duffy PA, Benvenisty N, Andrews PW, Ben-David U, et al. (2011) Assessing the safety of stem cell therapeutics. Cell Stem Cell 8: 618-628 [PubMed].

8. Lowry WE, Quan WL (2010) Roadblocks en route to the clinical application of induced pluripotent stem cells. J Cell Sci 123: 643-651 [PubMed].

9. Fong CY, Gauthaman K, Bongso A (2010) Teratomas from pluripotent stem cells: A clinical hurdle. J Cell Biochem 111: 769-781 [PubMed].

10. Kooreman NG, Wu JC (2010) Tumorigenicity of pluripotent stem cells: biological insights from molecular imaging. J R Soc Interface 6: S753-63 [PubMed].

11. Ben-David U, Benvenisty N (2011) The tumorigenicity of human embryonic and induced pluripotent stem cells. Nat Rev Cancer 11: 268-277 [PubMed].

12. Fong CY, Peh GS, Gauthaman K, Bongso A (2009) Separation of SSEA-4 and TRA-1-60 labelled undifferentiated human embryonic stem cells from a heterogeneous cell population using magnetic-activated cell sorting (MACS) and fluorescence-activated cell sorting (FACS). Stem Cell Rev 5: 72-80 [PubMed].

13. Malecki M, Hsu A, Truong L, Sanchez S (2012) Molecular immunolabeling with recombinant single-chain variable fragment (scFv) antibodies designed with metal-binding domains. Proc Natl Acad Sci U S A 99: 213-218 [PubMed].

14. Malecki M, Anderson M, Beauchaine M, Seo S, Tombokan X (2012) Maleck R. TRA $-1-60^{+}$, SSEA-4 $4^{+}$Oct $4 A^{+}$, Nanog ${ }^{+}$Clones of Pluripotent Stem Cells in Embryonal Carcinomas of the Ovaries. J Stem Cell Res Ther 2(5)130. [PubMed].

15. Malecki M, Tombokan X, Anderson M, Malecki R, Beauchaine M (2013) TRA- 
Citation: Malecki M, Vanne CL, Alhambra D, Dodivenaka C, Nagel S, et al. (2013) Safeguarding Stem Cell-Based Regenerative Therapy against latrogenic Cancerogenesis: Transgenic Expression of DNASE1, DNASE1L3, DNASE2, DFFB Controlled By POLA1 Promoter in Proliferating and Directed Differentiation Resisting Human Autologous Pluripotent Induced Stem Cells Leads to their Death. J Stem Cell Res Ther S9: 005e. doi:10.4172/2157-7633.S9-005 [PubMed]

1-60+, SSEA-4 $4^{+}$, POU5F1+, SOX2+ $2^{+}$Nanog ${ }^{+}$Clones of Pluripotent Stem Cells in Embryonal Carcinomas of the Testes. J Stem Cell Res Ther 3(1)134e:1-13 [PubMed].

16. Malecki M, Szybalski W. Isolation of single, intact chromosomes from single, selected ovarian cancer cells for in situ hybridization and next generation sequencing. Gene. 2012;493(1):132-139 [PubMed].

17. Tang C, Lee AS, Volkmer JP, Sahoo D, Nag D, et al. (2011) An antibody against SSEA-5 glycan on human pluripotent stem cells enables removal of teratomaforming cells. Nat Biotechnol 29: 829-834 [PubMed].

18. Lim DY, Ng YH, Lee J, Mueller M, Choo AB, et al. (2011) Cytotoxic antibody fragments for eliminating undifferentiated human embryonic stem cells. J Biotechnol 153: 77-85 [PubMed].

19. Choo AB, Tan HL, Ang SN, Fong WJ, Chin A, et al. (2008) Selection agains undifferentiated human embryonic stem cells by a cytotoxic antibody recognizing podocalyxin-like protein-1. Stem Cells 26: 1454-1463 [PubMed].

20. Ben-David U, Nudel N, Benvenisty N (2013) Immunologic and chemical targeting of the tight-junction protein Claudin- 6 eliminates tumorigenic human pluripotent stem cells. Nature Communications 4: 1992 [PubMed]

21. Schriebl K, Satianegara G, Hwang A, Tan HL, Fong WJ, et al. (2012) Selective removal of undifferentiated human embryonic stem cells using magnetic activated cell sorting followed by a cytotoxic antibody. Tissue Eng Part A 18: 899-909 [PubMed].

22. Ben-David U, Gan QF, Golan-Lev T, Arora P, Yanuka O, et al. (2013) Selective elimination of human pluripotent stem cells by an oleate synthesis inhibito discovered in a high-throughput screen. Cell Stem Cell 12: 167-179 [PubMed].

23. Cao F, Drukker M, Lin S, Sheikh AY, Xie X, et al. (2007) Molecular imaging of embryonic stem cell misbehavior and suicide gene ablation. Cloning Stem Cells 9: 107-117 [PubMed].

24. Rong Z, Fu X, Wang M, Xu Y (2012) A scalable approach to prevent teratoma formation of human embryonic stem cells. J Biol Chem 287: 32338-32345 [PubMed].

25. Cheng F, Ke Q, Chen F, Cai B, Gao Y, et al. (2012) Protecting against wayward human induced pluripotent stem cells with a suicide gene. Biomaterials 33 3195-3204 [PubMed].

26. Schuldiner M, Itskovitz-Eldor J, Benvenisty N (2003) Selective ablation of human embryonic stem cells expressing a "suicide" gene. Stem Cells 21: 257265 [PubMed].

27. Malecki M, Dahlke J, Haig M, Wohlwend L, Malecki R (2013) Eradication of ovarian cancer cells by transgenic expression of recombinant DNASE1, DNASE1L3, DNASE2, and DFFB controlled by EGFR promoter: Novel strategy for personalized therapy of cancer. J Genet Syndr Gene Ther 4(6)152e:1-10 [PubMed].
28. Tang HL, Yuen KL, Tang HM, Fung MC (2009) Reversibility of apoptosis in cancer cells. Br J Cancer 100: 118-122 [PubMed].

29. Deng W, Dai CL, Chen JJ, Kathawala RJ, Sun YL, et al. (2013) Tandutinib (MLN518) reverses multidrug resistance by inhibiting the efflux activity of the multidrug resistance protein 7 (ABCC10). Oncol Rep 29: 2479-2485 [PubMed]

30. Zhao WT, Wang YT, Huang ZW, Fang J (2013) BRCA2 affects the efficiency of DNA double-strand break repair in response to $\mathrm{N}$-nitroso compounds with differing carcinogenic potentials. Oncol Lett 5: 1948-1954 [PubMed].

31. Koller MR, Emerson SG, Palsson BO (1993) Large-scale expansion of human stem and progenitor cells from bone marrow mononuclear cells in continuous perfusion cultures. Blood 82: 378-384 [PubMed]

32. Ohnishi H, Oda Y, Aoki T, Tadokoro M, Katsube Y, et al. (2012) A comparative study of induced pluripotent stem cells generated from frozen, stocked bone marrow- and adipose tissue-derived mesenchymal stem cells. J Tissue Eng Regen Med 6: 261-271 [PubMed]

33. Malecki M, Malecki B, Malecki R (2011) Molecular death tags and methods of their use. WIPO Patents. WO 2012048161: 1-89 [WIPO].

34. Hu K, Yu J, Suknuntha K, Tian S, Montgomery K, et al. (2011) Efficient generation of transgene-free induced pluripotent stem cells from normal and neoplastic bone marrow and cord blood mononuclear cells. Blood 117: e109119 [PubMed]

35. Yu J, Hu K, Smuga-Otto K, Tian S, Stewart R, et al. (2009) Human induced pluripotent stem cells free of vector and transgene sequences. Science 324 797-801 [PubMed].

36. Narsinh KH, Jia F, Robbins RC, Kay MA, Longaker MT, et al. (2011) Generation of adult human induced pluripotent stem cells using nonviral minicircle DNA vectors. Nat Protoc 6: 78-88 [PubMed]

37. Malecki M (2013) Improved targeting and enhanced retention of the human autologous, fibroblast-derived, induced, pluripotent stem cells to the sarcomeres of the infarcted myocardium with the aid of the bioengineered, heterospecific, tetravalent antibodies. J Stem Cell Res Ther 3(2)138e:1-18 [PubMed]

38. Prasher DC, Eckenrode VK, Ward WW, Prendergast FG, Cormier MJ (1992) Primary structure of the Aequorea victoria green fluorescent protein. Gene 111 229-233 [PubMed].

39. Chalfie M, Tu Y, Euskirchen G, Ward WW, Prasher DC (1994) Green fluorescent protein as a marker for gene expression. Science 263: 802-805 [PubMed].

40. Heim R, Prasher DC, Tsien RY (1994) Wavelength mutations and posttranslational autooxidation of green fluorescent protein. Proc Natl Acad Sci USA 91: 12501-12504 [PubMed]

41. Heim R, Tsien R (1996) Engineering green fluorescent protein for improved brightness, longer wavelengths and fluorescence resonance energy transfer. Current Biology 6: 178-182 [PubMed]. 